\title{
The Fluoroscopic Technique for Monitoring Distraction of a Non-Invasive Lengthening Device in Early Onset Scoliosis
}

\author{
C. Thakar ${ }^{*}$, S. Sonecha, D. Rolton and C. Nnadi \\ Nuffield Orthopaedic Centre, Oxford University Hospitals NHS Trust, Windmill Road, Headington, Oxford, \\ Oxfordshire, UK
}

\begin{abstract}
Study Design: Prospective non-randomised.
Objective: To evaluate radiation exposure using fluoroscopy compared to x-rays in the monitoring of a growing rod system.

Background: The integrity of implants in the treatment of early onset scoliosis (EOS) is usually monitored with repeated plain x-rays. The cumulative dosage of ionizing radiation may be high. Children are highly sensitive to the carcinogenic effects of radiation and have a longer life span in which to reflect this risk. Fluoroscopy is commonly used to obtain focused images in other areas of orthopaedics. It has the advantage of less radiation intensity due to flash exposure. We have used fluoroscopy to monitor the lengthening of a non-invasive growing rod system in the treatment of EOS. We report on the results.

Methods: The technique was initially refined in vitro by performing an audit in which 10 radiographers screened a phantom spine (Plastic dummy) with an implanted non - invasive growing rod device. Pulse rate setting was 3 . Average exposure time was 0.01 seconds. Average radiation dose was $0.04 \mu \mathrm{Gym} 2$. A plain $\mathrm{x}$-ray of the same phantom spine yielded a radiation dose of $0.5 \mu \mathrm{Gym} 2$.

Radiation exposure using fluoroscopy was compared to that received using plain radiographs in ten consecutive patients treated for EOS. There were 8 males and 2 females. Average age 5.2 years.

Results: Mean radiation exposure using fluoroscopy was $0.0046 \mathrm{mSv}$ compared to an average radiation exposure of 0.115 $\mathrm{mSv}$ using standard $\mathrm{x}$-rays $(\mathrm{p}<0.003)$. Mean cancer induction risk was calculated to potentially reduce from 1:225,000 to 1:4.9 million.

Conclusion: We would recommend fluoroscopy as a useful technique to monitor the lengthening of a non-invasive growing rod system due to the significantly smaller radiation exposure.
\end{abstract}

Keywords: Early onset scoliosis, distraction, fluoroscopy, non-invasive growing rod, X-rays and radiation.

\section{BACKGROUND}

Non-invasive growing rod technology is gaining prominence as an alternative treatment option for early onset scoliosis. The principle advantage of this new technology is reducing the number of surgical episodes requiring general anaesthesia. However the integrity of these devices still needs to be monitored on a regular basis. Traditionally this has involved a child having as many as 4 sets of radiographs per year or even more if there has been revision surgery. Postero-anterior (PA) and Lateral (LAT) views are often required. Due to repeated imaging, the cumulative dosage of ionizing radiation may be high [1]. This is especially important in children, as they are highly sensitive to the carcinogenic effects of radiation and have a longer life span in which to reflect this risk. Fig. (1), from the Health Protection Agency (2011) [2] below shows the increased

*Address correspondence to this author at the Nuffield Orthopaedic Centre, Oxford University Hospitals NHS Trust, Windmill Road, Headington, Oxford, Oxfordshire, UK; Tel: +44 1865 741155; Fax: +44 1865 738027;

E-mail: chrishanthakar@gmail.com cancer risk secondary to radiation of the spine (lumbar and thoracic) at younger ages. The lifetime cancer risk of radiation at 5 years old is more than double that at 30 years old.

The amount of radiation in spinal radiographs is relatively high in comparison with radiographs of other areas. Typical effective doses of radiation in adult patients, from Health protection Agency, formerly known as National Radiological Protection Board (1998) are shown in Table 1 [3].

The International Commission for Radiological Protection (ICRP), Report 62 on "Radiological Protection in Biomedical Research" categorises radiation according to the level of risk (Table 2) [4].

This would potentially place spinal radiographs of children in the intermediate risk category (as paediatric patients have twice the lifetime cancer risk shown in the Table 1). Multiple radiographs, which are needed to monitor the distraction of the growing rods, would mean that the 


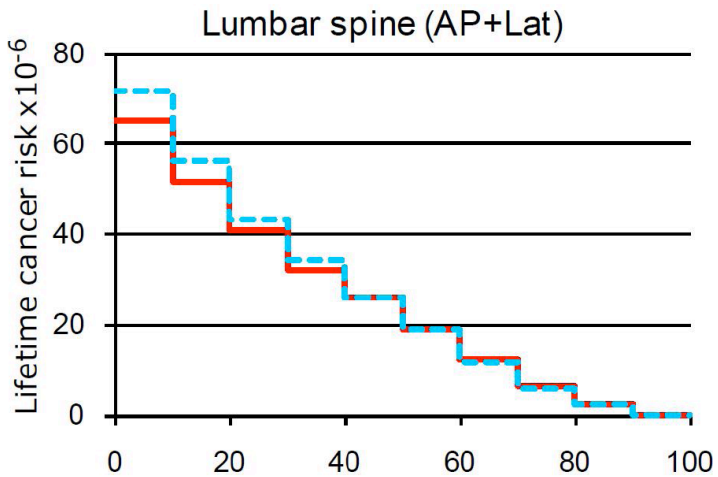

Age at exposure $(y)$

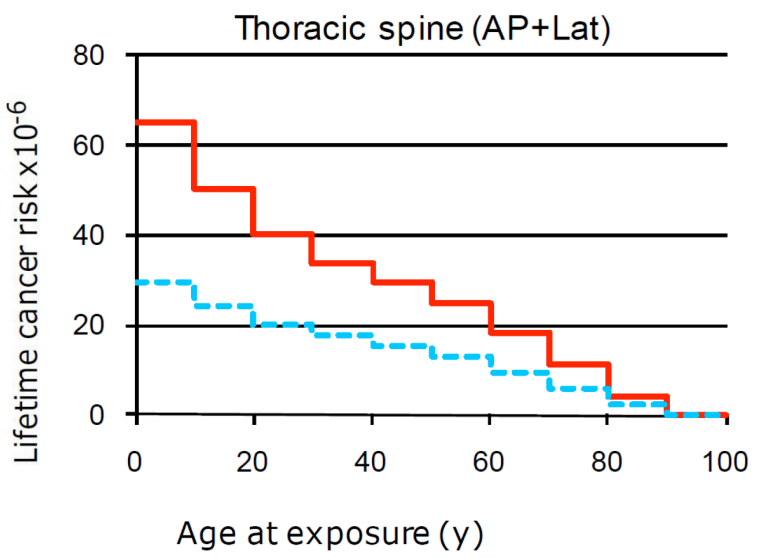

Fig. (1). Demonstrating lifetime cancer risk of lumbar and thoracic spine as a function of age of $\mathrm{x}$ ray radiation exposure where the red line represents females and blue line represents males, from the Health Protection Agency (2011) [2].

Table 1. Typical effective doses, equivalent periods of natural background radiation and lifetime fatal cancer risks from diagnostic medical exposures.

\begin{tabular}{|c|c|c|c|}
\hline Diagnostic Procedure & Typical Effective Dose (mSv) & $\begin{array}{l}\text { Equivalent Period of Natural } \\
\text { Background Radiation* }\end{array}$ & Risk of Fatal Cancer Per Examination** \\
\hline \multicolumn{4}{|l|}{$X$-ray examinations: } \\
\hline Limbs and joints (except hip) & $<0.01$ & $<1.5$ days & 1 in a few million \\
\hline $\begin{array}{l}\text { Teeth (single bitewing) } \\
\text { (panoramic) }\end{array}$ & $\begin{array}{c}<0.01 \\
0.01\end{array}$ & $\begin{array}{l}<1.5 \text { days } \\
1.5 \text { days }\end{array}$ & $\begin{array}{l}1 \text { in a few million } \\
1 \text { in } 2 \text { million }\end{array}$ \\
\hline Chest (single PA film) & 0.02 & 3 days & 1 in a million \\
\hline Skull & 0.07 & 11 days & 1 in 300,000 \\
\hline Cervical spine (neck) & 0.08 & 2 weeks & 1 in 200,000 \\
\hline Hip & 0.3 & 7 weeks & 1 in 67,000 \\
\hline Thoracic spine & 0.7 & 4 months & 1 in 30,000 \\
\hline Pelvis & 0.7 & 4 months & 1 in 30,000 \\
\hline Abdomen & 0.7 & 4 months & 1 in 30,000 \\
\hline Lumbar spine & 1.3 & 7 months & 1 in 15,000 \\
\hline Barium swallow & 1.5 & 8 months & 1 in 13,000 \\
\hline IVU (kidneys and bladder) & 2.5 & 14 months & 1 in 8000 \\
\hline Barium meal & 3 & 16 months & 1 in 6700 \\
\hline Barium follow & 3 & 16 months & 1 in 6700 \\
\hline Barium enema & 7 & 3.2 years & 1 in 3000 \\
\hline CT head & 2 & 1 year & 1 in 10,000 \\
\hline CT chest & 8 & 3.6 years & 1 in 2500 \\
\hline CT abdomen/pelvis & 10 & 4.5 years & 1 in 2000 \\
\hline \multicolumn{4}{|l|}{ Radionuclide studies: } \\
\hline Lung ventilation $\left({ }^{81 \mathrm{~m}} \mathrm{Kr}\right)$ & 0.1 & 2.4 weeks & 1 in 200,000 \\
\hline Lung perfusion $\left({ }^{99 \mathrm{~m}} \mathrm{Tc}\right)$ & 1 & 6 months & 1 in 20,000 \\
\hline Kidney $\left({ }^{99 \mathrm{~m}} \mathrm{Tc}\right)$ & 1 & 6 months & 1 in 20,000 \\
\hline Thyroid $\left({ }^{99 \mathrm{~m}} \mathrm{Tc}\right)$ & 1 & 6 months & 1 in 20,000 \\
\hline Bone $\left({ }^{99 \mathrm{~m}} \mathrm{Tc}\right)$ & 4 & 2.3 years & 1 in 5000 \\
\hline Dynamic cardiac $\left({ }^{99 \mathrm{~m}} \mathrm{Tc}\right)$ & 6 & 2.7 years & 1 in 3300 \\
\hline Myocardial perfusion $\left({ }^{201} \mathrm{Tl}\right)$ & 18 & 8 years & 1 in 1100 \\
\hline
\end{tabular}

*UK average $=2.2 \mathrm{mSv}$ per year: regional averages range from 1.5 to $7.5 \mathrm{mSv}$ per year.

**Approximate lifetime risk for patients from 16 to 69 years old: for paediatric patients multiply risks by about 2; for geriatric patients divide risks by about 5 . 
Table 2. Levels of radiaition exposure and cancer induction risk.

\begin{tabular}{|l|c|c|c|}
\hline \multicolumn{1}{|c|}{ Level of Risk } & Total Risk of Detrimental Radiation Effect & Effective Dose for Adults (mSv) & Level of Societal Benefit Needed \\
\hline \hline I. Trivial & $\sim 10^{-6}$ or less & $<0.1$ & Minor \\
\hline IIa. Minor & $\sim 10^{-5}$ & $0.1-1$ & Intermediate \\
\hline IIb. Intermediate & $\sim 10^{-4}$ & $1-10$ & Moderate \\
\hline III. Trivial & $\sim 10^{-3}$ or more & $>10$ & Substantial \\
\hline
\end{tabular}

radiation exposure would put these paediatric patients at moderate additional risk of lifetime cancer using the categories above. BEIR V (1989) suggested the risk of death from radiation induced cancer for a paediatric patient was $1.6 \%$ per $0.1 \mathrm{~Sv}$ and deduced there could be up to 2 deaths per 1000 scoliosis patient per $X$ ray [5].

Fluoroscopy provides real-time $\mathrm{X}$ ray imaging that is especially useful for guiding a variety of diagnostic and interventional procedures. It is commonly used to obtain focused images and involves flash exposure only. This greatly limits the amount of radiation exposure. A study by Geijer et al. found radiation doses in patients with scoliosis with digital exposure $(0.16 \mathrm{mSv})$ was ten times greater compared to that of fluoroscopy $(0.017 \mathrm{mSv})$ [1].

The use of fluoroscopy in the assessment of non-invasive growing rod technology has the theoretical benefits of reduced radiation dose and more precise evaluation of amount of lengthening achieved. As far as we are aware there have been no reports of the use of this technique in the literature. In this study, we have assessed the radiation exposure and cancer induction risk using fluoroscopy and compared it to the same risk if the conventional practice of $\mathrm{X}$-rays were to be continued.

\section{MATERIALS AND METHODS}

Ten consecutive patients have been evaluated for use of the fluoroscopy technique in assessing distraction and integrity of the implants. All procedures were performed at a single site by the senior author with a senior radiographer. Patients underwent their first lengthening three months following insertion of the non-invasive growing rod as part of their outpatient clinic appointment. No form of analgesia was required. Distraction was achieved using an external remote controller (ERC). The amount of distraction was calculated by the senior author based on predicted spinal growth [6].

An initial training session was undertaken to familiarise the radiographers with the fluoroscopic technique. A phantom spine was used (Plastic dummy of a child) to replicate bone and tissue. A non-invasive growing rod was placed on the phantom spine. This rod has an actuator which houses the lengthening mechanism. Any lengthening gap can be visualised in this region (Fig. 2).

\section{Fluoroscopy Technique}

The phantom was placed prone on a fluoroscopy table. The $\mathrm{C}$-arm was in the frontal position (image intensifier over the table, Fig. 3). Reference points were marked on the phantom's back to correspond to the actuator section of the rod.

Pulse rate setting was 3 . The $\mathrm{x}$-ray tube to image intensifier is a standard $80 \mathrm{~cm}$ apart. The beam is collimated with an average exposure of $60 \mathrm{Kvp}$ and 0.01 seconds duration.

Using these guidelines, 10 radiographers were asked to obtain images with fluoroscopy of the phantom spine and then acquire the same images of the phantom spine using plain x-rays. Average radiation dose using fluoroscopy was $0.04 \mu \mathrm{Gym} 2$. A plain $\mathrm{x}$-ray of the same phantom spine yielded a radiation dose of $0.5 \mu \mathrm{Gym} 2$.

\section{Digital Radiography Technique}

Done in PA and lateral positions. A pasting stand which is approximately 6 inches above the floor which the patient stands on is provided. The feet are slightly apart with the Chin almost horizontal to the floor and the arms extended.

The patient faces the Digital Receptor panel. The X-ray beam is set to incorporate the region from the top of the ear proximally to the greater trochanter distally.

The distance from the receptor panel to the patient's midline is fed into the digital machine (object to film distance).

The standard distance from beam tube to the receptor panel is $180 \mathrm{~cm}$. The beam is left wide open. Average automatic exposure is $70 \mathrm{Kvp}$ and 0.01 seconds duration.

Once the protocol had been established it was then applied to 10 consecutive patients who previously had a noninvasive growing rod implanted for treatment of EOS. All patients had at least 1 plain X-ray of their spines during the course of their growing rod treatment. The radiation dose from their most recent spine $\mathrm{x}$-ray was then compared to the radiation dose using the fluoroscopic technique. Each patient acted as its own control i.e., 1 set of PA and Lateral views versus 1 set of PA + Lateral views of the proximal and distal anchors along with 1 image of the actuator using fluoroscopy. In our centre the radiation dosage is registered on the imaging apparatus for each patient in real time.

\section{RESULTS}

The average radiation dose was $0.115 \mathrm{mSv}$ ( $\mathrm{sd} 0.0858$ ) with plain $\mathrm{x}$-ray. The average radiation dose with fluoroscopy was $0.0046 \mathrm{mSv}$ (sd 0.00334). Mean radiation exposure difference between the two imaging modalities was $0.1104 \mathrm{mSv}(\mathrm{p}<0.00296)$. The level of radiation exposure for each patient has been summarised in Table $\mathbf{3}$ along with 

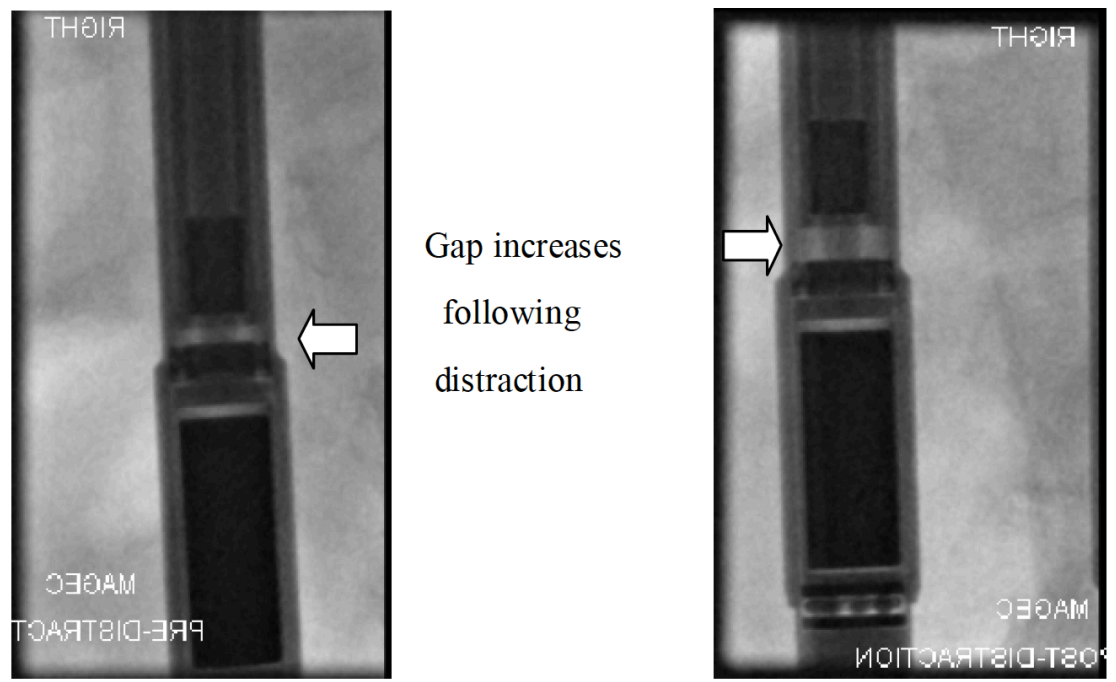

Fig. (2). Fluroscopy images demonstrating expansion of the non-invasive lengthening device. Pre and post distraction.

cancer induction risks based on the International Commission on Radiological Protection (ICRP 103, 2007) [7].

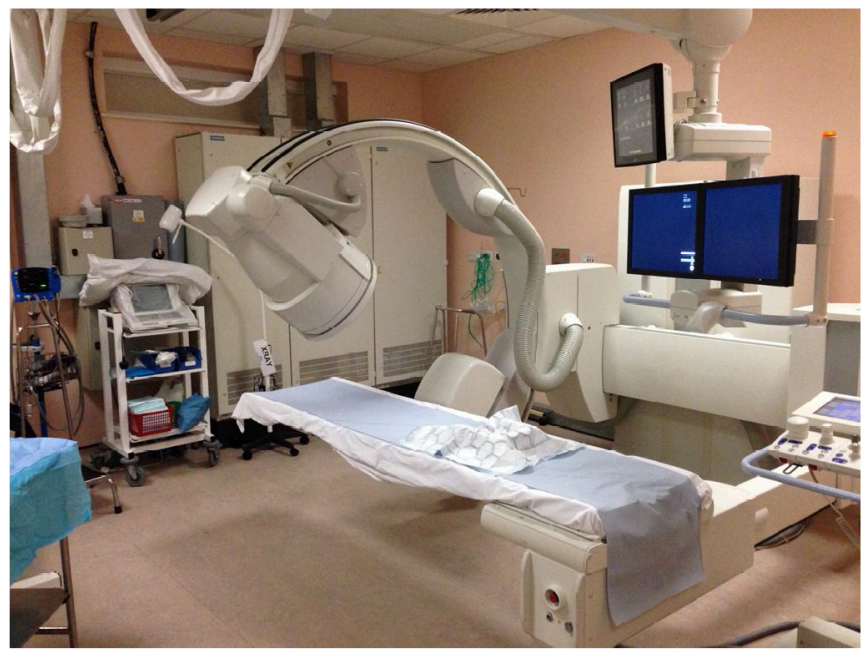

Fig. (3). Fluroscopy suite set up.

\section{DISCUSSION}

Management of early onset scoliosis is challenging. Traditional surgical treatment has involved the use of growing rods which need to be lengthened periodically. This requires follow up with repeated $\mathrm{x}$-rays to ensure treatment goals are being achieved and identify complications. Due to repeated imaging, the cumulative dosage of ionizing radiation may be high [1]. This principle is especially important in children, as they are highly sensitive to the carcinogenic effects of radiation and have a longer life span in which to reflect this risk.

We have reported a novel approach to the use of fluoroscopy for obtaining images in monitoring the lengthening of non-invasive growing rods in the treatment of early-onset scoliosis. This technique has the main advantage of a reduced effective dose of radiation. It drastically reduces the dose of radiation needed by as much as 40 fold. If the radiation dose from fluoroscopy in this study were compared to $\mathrm{x}$-ray doses recorded by the Health protection Agency (2011) [2] and NRPB (1998) [3] the 40 fold reduction would be considerably higher.

Table 3. Cohort results: comparison of levels of radiation exposure from radiographs versus fluoroscopy.

\begin{tabular}{|c|c|c|c|c|c|}
\hline Patient Number & Plain X-Ray (mSv) & Cancer Induction Risk & Fluoroscopy (mSv) & Cancer Induction Risk & Plain X-Ray/Fluoroscopy \\
\hline \hline 1 & 0.11 & 1 in 90000 & 0.005 & 1 in 2000000 & 25 \\
\hline 2 & 0.33 & 1 in 30000 & 0.005 & 1 in 1800000 & 60 \\
\hline 3 & 0.04 & 1 in 250000 & 0.013 & 1 in 700000 & 6 \\
\hline 4 & 0.04 & 1 in 250000 & 0.006 & 1 in 1500000 & 120 \\
\hline 5 & 0.18 & 1 in 50000 & 0.002 & 1 in 6000000 & 25 \\
\hline 6 & 0.07 & 1 in 140000 & 0.003 & 1 in 1800000 & 25 \\
\hline 7 & 0.12 & 1 in 75000 & 0.005 & 1 in 3000000 & 30 \\
\hline 8 & 0.09 & 1 in 100000 & 0.003 & 1 in 3000000 & 30 \\
\hline 9 & 0.08 & 1 in 100000 & 0.003 & 1 in 1000000 & 90 \\
\hline
\end{tabular}

Cancer induction risks are taken from ICRP 103, 2007 (whole population). 
A study by Geijer et al. [1] found radiation doses in scoliosis patients with digital exposure was $0.16 \mathrm{mSv}$, similar to the average radiation dose seen with pre-operative $\mathrm{x}$-rays in this study. The average radiation dose was $0.004 \mathrm{mSv}$ using fluoroscopy in our patients. This compares favourably to a study by Geijer et al. who reported a higher radiation dose of $0.017 \mathrm{mSv}$ using fluoroscopy. Compared to conventional plain radiographic techniques however, both studies demonstrate a drastic reduction in radiation dose when using fluoroscopy. However, in the study by Geijer et al. [1], fluoroscopic images were not localised and entailed 30-40 images stitched together. In this study, fluoroscopy was focused only on the anchor sites and actuator of the growing rod device which constituted a much smaller surface area. Hence the benefits of reduced radiation dose were more profound.

The average dose of radiation required for a single plain $\mathrm{x}$-ray is classified as a minor carcinogenic risk according to ICRP guidelines [3, 4]. However a minimum of 4 radiographs (AP and Lateral views) of the spine per year are needed to monitor growing rods. This would mean a much higher dose of radiation exposure. This potentially places these patients at moderate additional risk of lifetime cancer, using the IRCP guidelines (Table 2) [4]. In comparison, the radiation dose using the fluoroscopic technique would still be in the trivial carcinogenic risk category despite 4 sessions of exposure per year.

We have not encountered any complications using this technique but do recognise that there are some limitations to this technique and the study itself.

We have found that using fluoroscopy is effective in confirming and evaluating distraction of a non-invasive growing rod. The focused imaging taken at the distraction sites provides greater clarity that distraction has occurred and the degree of its extent compared to conventional x-rays. An additional benefit is that if required, distraction can be repeated and images retaken in the same sitting, minimising any potential distress to the child and parent. Imaging may also be used to check anchor points to ensure that there are no major complications with the construct. We have not assessed the clinical efficacy of fluoroscopy because this is not a randomised controlled trial. However, we believe it is an effective investigative tool which compares favourably with conventional plain radiographs. Visualisation of the whole spine and construct is possible with a reduction of magnification properties. This allows evaluation of the Cobb angle and integrity of the growing rod construct. The drawback is that the definition of the bony skeleton is not as clear as conventional $\mathrm{x}$-rays but for the purposes of monitoring the construct it was far superior.

We have not found fluoroscopy to be more difficult than conventional $\mathrm{x}$-rays to perform and the risk of radiation exposure to both parents and radiographer are minimised by taking standard precautions, wearing lead gowns and standing behind a lead screen. Given that fluoroscopy uses less radiation, the risk to both parents and radiographer is small compared to conventional $\mathrm{x}$-rays. Additionally, fluoroscopy uses focused beams with very minimal scatter. All our radiographers have been trained in the use of this technique. We have not noticed an increase in procedure time when compared to conventional $\mathrm{x}$-rays. Using a phantom spine first, enabled us to safely establish the appropriate level of exposure to ensure adequate imaging of the rod and spine. If the quality of the images is poor, the image may be retaken with significantly less radiation exposure compared to repeating another conventional $\mathrm{x}$-ray. Additionally, this can be repeated in the same sitting, reducing any anxiety to the child and parent.

With regards to the study itself, the sample size is small and there was no control group for comparison although a phantom spine was used to establish the settings required for satisfactory images. We also acknowledge the practical constraints determined by local practice with regards to accessibility to fluoroscopy suites. Performing this technique in the outpatient setting may be challenging and does require a greater degree of co-operation by the child initially but this is usually minimal and eased by allowing the parent of the child to be present in the fluoroscopy suite.

Despite these limitations, the benefits of the technique are still evident by the significance in radiation exposure reduction. Additionally, the benefits of a group approach to distractions performed in a dedicated clinic session cannot be underestimated. It is reassuring to parents and children to share their experiences with other families in a similar situation and helps to demystify the distraction process. The waiting area in our children's x-ray department is often the venue for new found friendships and shared experiences.

\section{CONCLUSION}

The study has shown that fluoroscopy can be used to monitor growing rod devices in children with early onset scoliosis. More importantly, the radiation exposure from this technique compared to plain $\mathrm{x}$-rays is significantly less. The lifetime carcinogenic risk is therefore reduced in these children. We would recommend this technique as a very useful alternative to plain $\mathrm{x}$-rays in settings where this facility is available.

\section{CONFLICT OF INTEREST}

The authors confirm that this article content has no conflict of interest.

\section{ACKNOWLEDGEMENTS}

Declared none.

\section{REFERENCES}

[1] Geijer H, Beckman K, Jonsson B, Andersson T, Persliden J. Digital radiography of scoliosis with a scanning method: initial evaluation. Radiology 2001; 218(2): 402-10.

[2] Wall BF, Haylock R, Jansen JTM, Hillier MC, Hart D, Shrimpton PC. Medical risks of $x$ ray examinations as a function of age and sex. Health Protection Association 2001; pp. 1-52.

[3] Health Protection Association 2008. Available from: http://www.hp a.org.uk/Topics/Radiation/Unders

tandingRadiation/UnderstandingRadiationTopics/MedicalRadiation s/medic TedEquivalent/..

[4] The international commission of radiological Protection (IRCP). Radiological Protection in Biomedical Research- report 62. Ann ICRP 1992; 22(3). 
[5] National Research Council, Committee on the Biological Effects of Ionizing Radiations (BEIR V), Health effects of exposure to low levels of ionizing radiation. Washington, DC: Natl Acad Press 1990.
[6] Dimeglio A. Growth in Pediatric Orthopaedics. J Paed Orth 2001; 21: 549-55.

[7] The 2007 Recommendations of the International Commission on Radiological Protection (ICRP). Ann ICRP 2007; 37(2-4): 1-332.

Received: December 24, 2013

Revised: March 28, 2014

Accepted: May 7, 2014

(C) Thakar et al.; Licensee Bentham Open.

This is an open access article licensed under the terms of the Creative Commons Attribution Non-Commercial License (http://creativecommons.org/licenses/by$\mathrm{nc} / 3.0 /$ ) which permits unrestricted, non-commercial use, distribution and reproduction in any medium, provided the work is properly cited. 